\title{
National Electrical Manufacturers Association and Clinical Evaluation of a Novel Brain PET/CT Scanner
}

\author{
Kira S. Grogg ${ }^{1}$, Terrence Toole ${ }^{2}$, Jinsong Ouyang ${ }^{1}$, Xuping Zhu ${ }^{1}$, Marc D. Normandin ${ }^{1}$, Quanzheng Li $^{1}$, Keith Johnson ${ }^{3}$, \\ Nathaniel M. Alpert ${ }^{1}$, and Georges El Fakhri ${ }^{1}$ \\ ${ }^{I}$ The Gordon Center for Medical Imaging, Department of Radiology, Massachusetts General Hospital/Harvard Medical School, \\ Boston, Massachusetts; ${ }^{2}$ Photo Diagnostic Systems, Inc., Boxborough, Massachusetts; and ${ }^{3}$ Division of Nuclear Medicine and \\ Molecular Imaging, Department of Radiology, Massachusetts General Hospital/Harvard Medical School, Boston, Massachusetts
}

The aim of this study was to determine the performance of a novel mobile human brain/small-animal PET/CT system. The scanner has a 35.7-cm-diameter bore and a $22-\mathrm{cm}$ axial extent. The detector ring has 7 modules each with $3 \times 4$ cerium-doped lutetium yttrium orthosilicate crystal blocks, each consisting of $22 \times 22$ outer-layer and $21 \times$ 21 inner-layer crystals, each layer $1-\mathrm{cm}$ thick. Light is collected by $12 \times 12$ silicon photomultipliers. The integrated CT can be used for attenuation correction and anatomic localization. The scanner was designed as a low-cost device that nevertheless produces high-quality PET images with the unique capability of battery-powered propulsion, enabling use in many settings. Methods: Spatial resolution, sensitivity, and noise-equivalent counting rate were measured based on the National Electrical Manufacturers Association NU2-2012 procedures. Reconstruction was done with tight energy and timing cuts-400-650 keV and $7 \mathrm{~ns}$ - and loose cuts-350-700 keV and $10 \mathrm{~ns}$. Additional image quality measurements were made from phantom, human, and animal studies. Performance was compared with a reference scanner with comparable imaging properties. Results: The full width at half maximum transverse resolution at a $1-\mathrm{cm}(10-\mathrm{cm})$ radius was $3.2 \mathrm{~mm}$ (5.2- $\mathrm{mm}$ radial, $3.1-\mathrm{mm}$ tangential), and the axial resolution was $3.5 \mathrm{~mm}(4.0 \mathrm{~mm})$. A sensitivity of 7.5 and $11.7 \mathrm{kcps} / \mathrm{MBq}$ at the center for tight and loose cuts, respectively, increased to 8.8 and $13.9 \mathrm{kcps} / \mathrm{MBq}$, respectively, at a $10-\mathrm{cm}$ radial offset. The maximum noise-equivalent counting rate of 19.5 and $22.7 \mathrm{kcps}$ for tight and loose cuts, respectively, was achieved for an activity concentration of $2.9 \mathrm{kBq} / \mathrm{mL}$. Contrast recovery for $4: 1$ hot cylinder to warm background was $76 \%$ for the $25-\mathrm{mm}$-diameter cylinder but decreased with decreasing cylinder size. The quantitation agreed within $2 \%$ of the known activity distribution and concentration. Brain phantom and human scans have shown agreement in SUVs and image quality with the reference scanner. Conclusion: We characterized the performance of the NeuroPET/CT and showed images from the first human studies. The study shows that this scanner achieves good performance when spatial resolution, sensitivity, counting rate, and image quality along with a low cost and unique mobile capabilities are considered.

Key Words: PET/CT; instrumentation; NEMA standard

J Nucl Med 2016; 57:646-652

DOI: 10.2967/jnumed.115.159723

\footnotetext{
Received Jun. 23, 2015; revision accepted Nov. 25, 2015.

For correspondence or reprints contact: Georges El Fakhri, Massachusetts General Hospital, 55 Fruit St., Boston, MA 02114.

E-mail: elfakhri.georges@mgh.harvard.edu

Published online Dec. 23, 2015.

COPYRIGHT @ 2016 by the Society of Nuclear Medicine and Molecular Imaging, Inc.
}

$\mathbf{P}$

ET has proved to be a powerful tool for diagnosis of dementia (1) and diagnosis and monitoring of brain tumors (2). It can also be used to measure responses to stimuli. Renewed interest in brain imaging is leading to the development of dedicated head scanners with a low cost while still maintaining high sensitivity and spatial resolution.

The NeuroPET/CT is a full-ring mobile brain PET/CT scanner developed by Photo Diagnostic Systems, Inc. $(3,4)$. It is the firstever dedicated brain PET/CT scanner. Although developed primarily for brain imaging, it can also be used for animal and pediatric imaging. Because all PET data are acquired in list-mode, it is easy to create dynamic sequences and reconstruction. The NeuroPET/CT is self-propelled on battery-powered wheels, such that it can be driven to multiple locations. Because of its mobility and ability to use any standard power outlet, the NeuroPET/CT can be used in a flexible manner. Instead of a motorized bed moving through the bore, as with typical scanners, the NeuroPET/CT moves on treads to collect CT data and can be used with any bed that can be adjusted to the appropriate height. A NeuroPET/CT scanner was installed at Massachusetts General Hospital (MGH) in 2011. After a period of studies on its performance, the scanner is now used for clinical and preclinical research studies. Figure 1 shows a picture of the scanner with major components labeled.

We evaluated the imaging characteristics of the PET component of the NeuroPET/CT scanner using the National Electrical Manufacturers Association (NEMA) standards measurements (5), and with additional phantom, animal, and human studies.

\section{MATERIALS AND METHODS}

\section{PET Specifications}

NeuroPET/CT was designed to achieve high sensitivity and good spatial resolution. The PET detector consists of 1-cm-long ceriumdoped lutetium yttrium orthosilicate crystals divided into modules and blocks. The physical specifications of the NeuroPET/CT are summarized in Table 1, along with specifications for the ECAT Exact HR+ scanner (Siemens), which is currently used at MGH for brain imaging. Event positions are determined using the centroid from the silicon photomultiplier array on each block. Although the offset inner and outer crystal layers will eventually be used to determine the depth of interaction, they are currently treated as a single layer with a single characteristic conversion depth. The 22-cm axial extent of the PET rings allows the human brain to be imaged in a single acquisition.

PET data are taken in list-mode, and reconstruction software creates sinograms with randoms, scatter, and attenuation corrections 


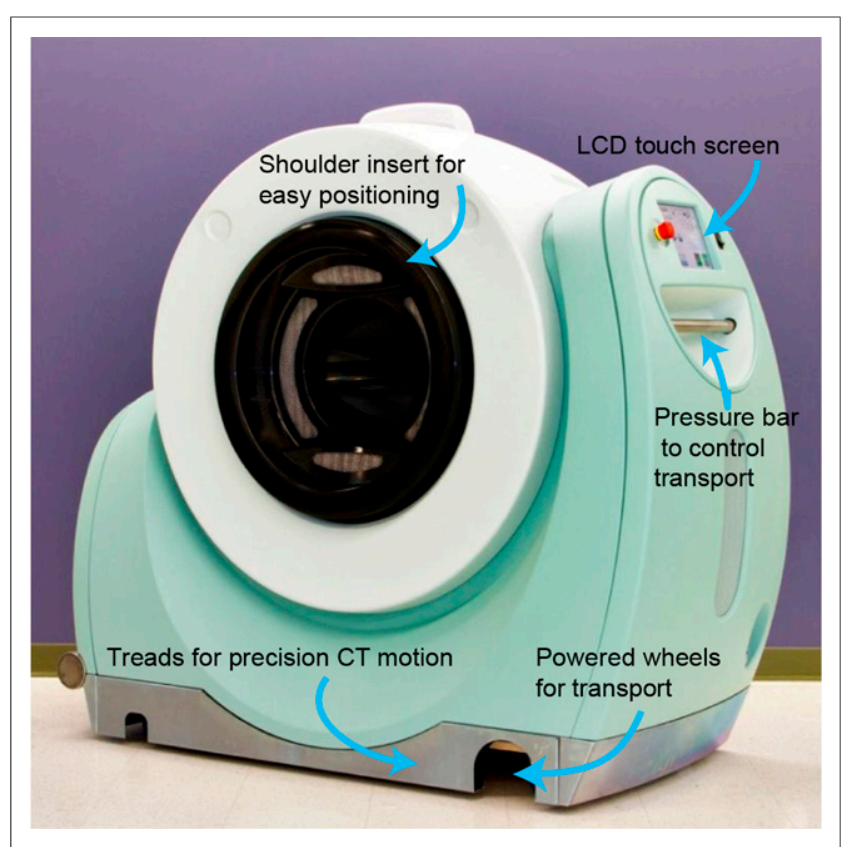

FIGURE 1. NeuroPET/CT scanner with outer components labeled. Treads (behind metal apron) are used for precise scanner motion during CT scans, whereas wheels are used to transport scanner to scan location.

for multiple temporal frames. A dead-time correction is applied on the basis of a paralyzable model at the block level. Singles rates for each block are collected every $6 \mathrm{~s}$ during an acquisition and used in the dead-time correction. Delayed data used for the randoms correction have offline cuts and live fraction applied before sinogram binning. Smoothing is applied before subtraction from the prompts. Radioactive decay is corrected on a per-frame basis. For scatter correction, the standard single scatter simulation method (6) is used. To correct for inhomogeneity and variations in the coincidence channels (i.e., lines of response), a 2-h normalization scan is taken of a thin (low scatter) 30.5-cm-long annulus with an inner diameter of $27.9 \mathrm{~cm}$ and an outer diameter of $29.2 \mathrm{~cm}$ filled with approximately $18 \mathrm{MBq}$ of ${ }^{18} \mathrm{~F}$. The data are binned into sinograms, and randoms are subtracted. Normalization data are corrected for differences in the activity distribution and are variance-reduced before being used as a normalization correction during reconstruction. Attenuation correction is done using a low-dose CT scan taken with each PET scan. The bilinear scaling method is used to convert CT images in Hounsfield units to $\mu$ values at $511 \mathrm{keV}(7,8)$.

Iterative maximum likelihood-expectation maximum and filtered backprojection are reconstruction options. Reconstructions are done in 2 dimensions after Fourier rebinning (9). The projection operator is a line integral calculated on the fly, for which the integrand is determined by performing a bilinear interpolation in image space at fixed intervals along the line. The reconstruction uses a maximum ring difference that is half of the axial field of view (FOV). The hardware coincidence time window is $10 \mathrm{~ns}$ but can be reduced in the software. Similarly, the energy window can be set to anywhere between 350 and $750 \mathrm{keV}$. Within this paper, tight cuts refers to an energy window of $400-650 \mathrm{keV}$ and a timing window of $7 \mathrm{~ns}$. Loose cuts refers to an energy window of 350-700 keV and a timing window of $10 \mathrm{~ns}$. The reconstructions in this paper use $256 \times 256 \times 92$ image space bins for $1 \times 1 \times 2.3 \mathrm{~mm}$ voxels or $256 \times 256 \times 184$ image space bins for $1 \times 1 \times$ $1.17 \mathrm{~mm}$ voxels. Images are calibrated to radioactivity concentrations in $\mathrm{Bq} / \mathrm{mL}$ using a 6-L cylindric head-sized source of known activity concentration.

\section{CT Specification and Safety Assessment}

The CT has 3,264 detector channels with 8 axial slices at spacing of about $1.25 \mathrm{~mm}$. The $\mathrm{x}$-ray source, capable of 100,120 , or $140 \mathrm{kVp}$ at 2.0-7.0 mA, can rotate at $60 \mathrm{rpm}$ for 1,440 views/s. The system is capable of taking full axial FOV helical CTs in under $15 \mathrm{~s}$. Low-dose CT scans deliver a dose of less than 100 mrem.

The limiting factor for the mobility of the NeuroPET/CT is the external radiation produced during CT operation. Scatter dose rates were measured with a 20 -m-diameter, $27-\mathrm{cm}$-length cylindric water phantom at the CT isocenter. Rates were measured for low-dose CT scans (2-mA tube current) and higher-dose, higher-quality, scans (7 mA). All scans were $220 \mathrm{~mm}$ axially at $120 \mathrm{kVp}$, with a 2 -s resolution. At $1.5 \mathrm{~m}$ from the scanner and at $1.2-\mathrm{m}$ height, the maximum dose received at a $45^{\circ}$ angle from patient axis is 308 and 1,076

TABLE 1

Design Characteristics of NeuroPET/CT with HR+ for Comparison

\begin{tabular}{|c|c|c|}
\hline Characteristic & NeuroPET/CT value & $\mathrm{HR}+$ value \\
\hline Detector ring diameter $(\mathrm{cm})$ & 35.7 & 82.4 \\
\hline Detector material & LYSO:Ce & BGO \\
\hline No. of crystals & 77,700 & 18,432 \\
\hline Modules & 7 & 32 rings \\
\hline Blocks/module & $3 \times 4$ & \\
\hline Crystal size (mm) & $2.3 \times 2.3 \times 10(\times 2$ layers $)$ & $4.05 \times 4.39 \times 30$ \\
\hline Crystal array/block & $22 \times 22$ outer, $21 \times 21$ inner & 16 crystals/pmt \\
\hline Transaxial FOV $(\mathrm{cm})$ & 25 & 58.3 \\
\hline Axial FOV $(\mathrm{cm})$ & 22 & 15.5 \\
\hline Coincidence window (ns) & Online, 10.14 (offline, 7) & 12 \\
\hline Energy window (keV) & Variable, default: 400-650 & $300-650$ \\
\hline
\end{tabular}


$\mu$ rem for a low- and high-dose, respectively, scan. The worst-case exposure at $3 \mathrm{~m}$, with the scanner running at the highest dose settings, is 140 (470) $\mu$ rem.

\section{PET Performance}

Spatial Resolution. The spatial resolution was measured using 3 plastic-encased $0.19-\mathrm{MBq}(5-\mu \mathrm{Ci}){ }^{22} \mathrm{Na}$ point sources less than 1 $\mathrm{mm}$ in all dimensions. The point sources were placed at 2 axial positions, the center of the FOV, and $8.5 \mathrm{~cm}$ from the center and scanned for $15 \mathrm{~min}$ each. For each axial location, the sources were placed at $3(x, y)$ positions: $(1 \mathrm{~cm}, 0),(0,10 \mathrm{~cm})$, and $(10 \mathrm{~cm}, 0)$. The data were reconstructed using filtered backprojection with $1 \times$ $1 \times 1.17 \mathrm{~mm}$ voxel size, tight cuts, and no spatial smoothing. A parabolic fit was used to find the peak, and then linear interpolation was used to find the full width at half maximum and the full width at tenth maximum of the point-spread functions in all 3 cardinal directions, which were combined into radial, transverse, and axial results.

Sensitivity. The sensitivity was measured with a standard set of thin aluminum sleeves around a $70-\mathrm{cm}$ line source filled with 6.81 $\mathrm{MBq}$ at the start of acquisition, resulting in negligible dead time. The line source was placed in the center of the transverse FOV, parallel to the scanner bore. Five aluminum sleeves with increasing inner diameter and constant wall thickness were added to the line source, and a 180-s acquisition was taken for each additional sleeve. Sinograms were corrected for decay and randoms, and data were single slice rebinned (10). The sensitivity was calculated as the normalized sum of the corrected activity for each acquisition. Counting rates were plotted against accumulated aluminum thickness. The absolute sensitivity was obtained by extrapolating the data to zero thickness (no attenuation) using linear regression. The analysis was performed with both tight and loose cuts. The procedure was repeated but with the setup at 5 and $10 \mathrm{~cm}$ from the center of the FOV.

Noise Equivalent Counting Rate (NECR). The NECR is the ratio of the square of the true rate to the total rate (true + random + scatter): $T^{2} /(T+R+S)$. It is proportional to the square of the signal-to-noise ratio in the reconstructed images. A 20 -cm-diameter polyethylene scatter cylinder $\left(\rho=0.96 \mathrm{~g} / \mathrm{cm}^{3}\right)$ with a length of $70 \mathrm{~cm}$ was used to measure the NECR. A plastic tube was placed through the full length of the cylinder at $4.5 \mathrm{~cm}$ from the center and filled with $173 \mathrm{MBq}$ of ${ }^{18} \mathrm{~F}$ at the scan start. The cylinder was centered axially and transaxially in the scanner with the line source closest to the table.

The data were acquired over a $24-\mathrm{h}$ period as 20 frames of increasing length, from $79 \mathrm{~s}$ to $1 \mathrm{~h}$, each separated by $45 \mathrm{~min}$. A blank scan was taken $44 \mathrm{~h}$ later, with the phantom in place but no remaining activity, to measure the intrinsic lutetium yttrium orthosilicate background counting rate. Sinograms were created for each frame using both tight and loose cuts. The sinograms were analyzed according to the NEMA instructions, and noise equivalent count event rates were calculated for each acquisition.

Scatter Fraction $(S F)$. The SF was calculated with NECR data based on the methods described by Watson et al. (11). For each activity concentration, $i$, the scatter fraction, SF, was calculated according to the equation $\mathrm{SF}_{\mathrm{i}}=\mathrm{R}_{\text {scatter }} /\left(\mathrm{R}_{\text {true }}+\mathrm{R}_{\text {scatter }}\right)$, where $R_{\text {scatter }}$ was calculated by subtracting the delayeds estimated random event rate, the trues rate $R_{\text {true }}$, and the intrinsics trues rate (measured from the blank scan) from the total rate calculated within a $12-\mathrm{cm}$ radius.

Dead-Time Correction. The NECR measurement data were used to assess the dead-time correction by comparing the system singles rate with and without dead-time corrections versus activity concentration.

\section{Image Quality and Quantitation}

Uniform Phantoms. A uniform cylindric water phantom of volume $6,283 \mathrm{~mL}$ was filled with $20 \mathrm{MBq}$ of ${ }^{18} \mathrm{~F}$ and scanned for $15 \mathrm{~min}$. The data were reconstructed with tight cuts. An aliquot was used to measure the activity concentration with a well counter to compare with the image concentration. Images were examined for uniformity in axial and transaxial directions.

A contiguous grid of $10 \times 10 \mathrm{~mm}$ regions of interest (ROIs), all contained within a circle of radius of $88 \mathrm{~mm}$, was created for each slice. As a measure of concentration variability, a coefficient of variation was determined by calculating the SD of the means of the ROI counts, normalized by the mean of all ROI within each slice.

American College of Radiology Phantom. An American College of Radiology accreditation phantom, with a section of wedges of cold rods of varying sizes $(4.8,6.4,7.9,9.5,11.1$, and $12.7 \mathrm{~mm})$, a uniform section, and a section with cold and hot cylinders, was filled with ${ }^{18} \mathrm{~F}$ such that the hot cylinders-to-background ratio was $4: 1$. The total activity of about $13 \mathrm{MBq}$ corresponds to that expected from a $222-\mathrm{MBq}$ injection to a $70-\mathrm{kg}$ patient. The phantom was scanned for $15 \mathrm{~min}$ and reconstructed with tight cuts. The cold and hot cylinders were compared with the background activity to measure contrast recovery. The same phantom, fill, and scan parameters were performed on the $\mathrm{HR}+$, and the data were reconstructed using standard brain imaging settings (described below) but without the usual segmenting of the attenuation map, to avoid artifacts from overcorrection of air pockets.

The contrast recovery coefficients for the hot cylinders were calculated as $\mathrm{CRC}=(\mathrm{H} / \mathrm{B}-1) /(\mathrm{a}-1)$, where $\mathrm{H}$ is the mean hot concentration in a single-slice ROI with diameter corresponding to each cylinder diameter, $\mathrm{B}$ is the background concentration estimated from 60 ROIs of the same size as the corresponding cylinder, and a is the true hot-to-background ratio. The cold cylinders' recovery coefficients were calculated as $(\mathrm{B}-\mathrm{C}) / \mathrm{B}$, where $\mathrm{C}$ is the mean cold cylinder concentration.

Hoffman Brain Phantom. ${ }^{18} \mathrm{~F}-\mathrm{FDG}$ was added to a water-filled Hoffman 3-dimensional brain phantom that was then shaken for several minutes and allowed to mix for another $2 \mathrm{~h}$. A 15-min PET scan on the NeuroPET/CT was started when the phantom reached an activity of $27 \mathrm{MBq}$, slightly lower than the typical activity present in the FOV during an ${ }^{18} \mathrm{~F}-\mathrm{FDG}$ scan. The same procedure was later repeated on the $\mathrm{HR}+$. Both the $\mathrm{HR}+$ and the NeuroPET/CT images were reconstructed with the same respective parameters as for typical brain studies. For comparison purposes, the NeuroPET/CT images were rigidly registered to the $\mathrm{HR}+$ images by minimizing the least-squares difference in image intensities.

Human Scans. Over 50 human subjects have been scanned using the NeuroPET/CT at MGH. Each subject was injected with $190 \pm 10$ $\mathrm{MBq}$ of ${ }^{18} \mathrm{~F}$-FDG. After $47 \pm 6 \mathrm{~min}$ of uptake, the subject was scanned first on an HR + for $15 \mathrm{~min}$ and then $14 \pm 6$ min later on the NeuroPET/CT for a 15-min PET. All human studies were approved by the MGH institutional review board, and written informed consent was obtained.

The HR + images were reconstructed as a single frame using the standard brain imaging settings: ordered-subset expectation maximum algorithm with 3 iterations, 16 subsets, 2-mm gaussian smoothing, and a voxel size of $2 \times 2 \times 2.4 \mathrm{~mm}$. The NeuroPET/CT images were reconstructed with maximum likelihood-expectation maximum, 100 iterations, $1.25-\mathrm{mm}$ inter-iteration gaussian smoothing, 1.85 -mm gaussian postsmoothing, a voxel size of $1 \times 1 \times 2.3 \mathrm{~mm}$, and the tight set of cuts.

Thirty-three subjects scanned on the NeuroPET/CT and the HR+, and who also had undergone an MR scan, were selected for analysis. 
TABLE 2

NeuroPET/CT and HR + Spatial Resolutions from Point Sources

\begin{tabular}{|c|c|c|c|c|c|}
\hline \multirow{3}{*}{$\begin{array}{l}\text { Full width } \\
\text { at half and tenth } \\
\text { maximum }(\mathrm{mm})\end{array}$} & \multicolumn{5}{|c|}{ Radial position } \\
\hline & \multicolumn{2}{|l|}{$1 \mathrm{~cm}$} & \multicolumn{3}{|c|}{$10 \mathrm{~cm}$} \\
\hline & Transverse & Axial & Radial & Tangential & Axial \\
\hline FWHM NP & 3.2 & 3.5 & 5.2 & 3.1 & 4.0 \\
\hline FWHM HR+ & 4.5 & 5.1 & 6.8 & 4.8 & 6.2 \\
\hline FWTM NP & 6.0 & 6.8 & 8.3 & 5.5 & 8.3 \\
\hline FWTM HR+ & 8.2 & 11.2 & 12.2 & 9.9 & 13.5 \\
\hline
\end{tabular}

$\mathrm{NP}=$ NeuroPET/CT; FWHM = full width at half maximum; FWTM $=$ full width at tenth maximum.

${ }^{18}$ F-FDG PET image volumes were registered to each subject's MR image using the rigid-body registration algorithms in the statistical parametric mapping software (Wellcome Trust Centre for Neuroimaging) (12). The MR volume was then registered to the ICBM-152 MNI brain template (13) using nonlinear registration. The resulting registration matrix was used to transform the 2 corresponding PET image volumes. The average SUV within each of 9 anatomic regions (caudate nucleus, cerebellum, frontal lobe, insular cortex, occipital lobe, parietal lobe, putamen, temporal lobe, and thalamus) was computed for each subject using the MNI structural atlas. A scatterplot to show the relationship between the measured SUVs on the HR + and NeuroPET/CT was made for all 9 regions and 33 subjects.

To establish the linear relation between SUVs measured on these scanners, the within-patient SUVs from the 2 scanners were fitted to the linear model with blocked total least squares (TLS) regression (14):

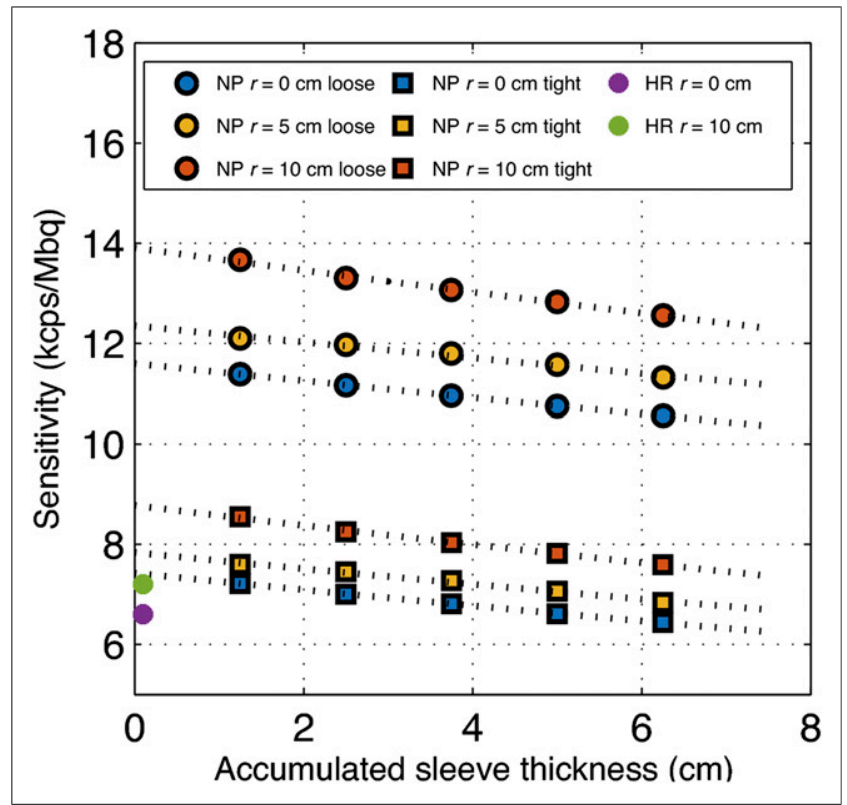

FIGURE 2. Sensitivity as function of sleeve thickness for 3 different radial placements on the NeuroPET/CT (NP). Lower HR+ sensitivity is shown for reference. $\bullet=$ Loose cuts; $\bullet$ = tight cuts.

$$
\operatorname{SUV}_{\mathrm{NP} / \mathrm{CT}}(\mathrm{i}, \mathrm{j})=\mathrm{k} \mathrm{SUV}_{\mathrm{HR}}+(\mathrm{i}, \mathrm{j})+\mathrm{C}_{\mathrm{i}},
$$

where subscript $\mathrm{i}$ denotes subject and subscript $\mathrm{j}$ denotes region. This analysis yields a mutual regression slope, $\mathrm{k}$, for all subjects and an intercept, $\mathrm{C}_{\mathrm{i}}$, for each subject that accounts for subjectspecific uptake and clearance rates. TLS was also performed for each subject separately, and the mean and SD of the slopes were calculated.

Animal Scan. In addition to the human brain studies, a low-dose ${ }^{11} \mathrm{C}$ kinase tracer study was performed on a macaque monkey. The monkey was injected with $9.25 \mathrm{MBq}(250 \mu \mathrm{Ci})$ at the start of PET acquisition and scanned for $15 \mathrm{~min}$, and images were reconstructed using the tight cuts with $1 \times 1 \times 1.17 \mathrm{~mm}$ voxels. The $\mathrm{MGH}$ animal care committee approved all animal studies.

\section{RESULTS}

\section{Spatial Resolution}

The spatial resolution for each of the point source measurements is listed in Table 2 along with literature-based values for the HR $+(15,16)$ that were calculated using the same analysis method.

\section{Sensitivity}

Figure 2 shows the results of the sensitivity as a function of accumulated sleeve thickness for the NeuroPET/CT and the sensitivity of the HR+. The center of the FOV has a sensitivity of almost $0.75 \%$ (i.e., $7.5 \mathrm{kcps} / \mathrm{MBq}$ ), increasing to $0.88 \%$ at a $10-\mathrm{cm}$ offset. With the looser energy and timing windows, the sensitivity increased to $1.16 \%(1.39 \%)$ at the center (radial) placement. The sensitivity of the HR + is much smaller $-0.66 \%$ central and $0.72 \%$ at $10-\mathrm{cm}$ offset.

\section{NECR}

Figure 3 shows the NECR versus activity concentration. With tight cuts, the maximum NECR of $19.5 \mathrm{kcps}$ was achieved for an activity concentration of $2.9 \mathrm{kBq} / \mathrm{mL}$. With the loose cuts, the peak NECR was $22.7 \mathrm{kcps}$.

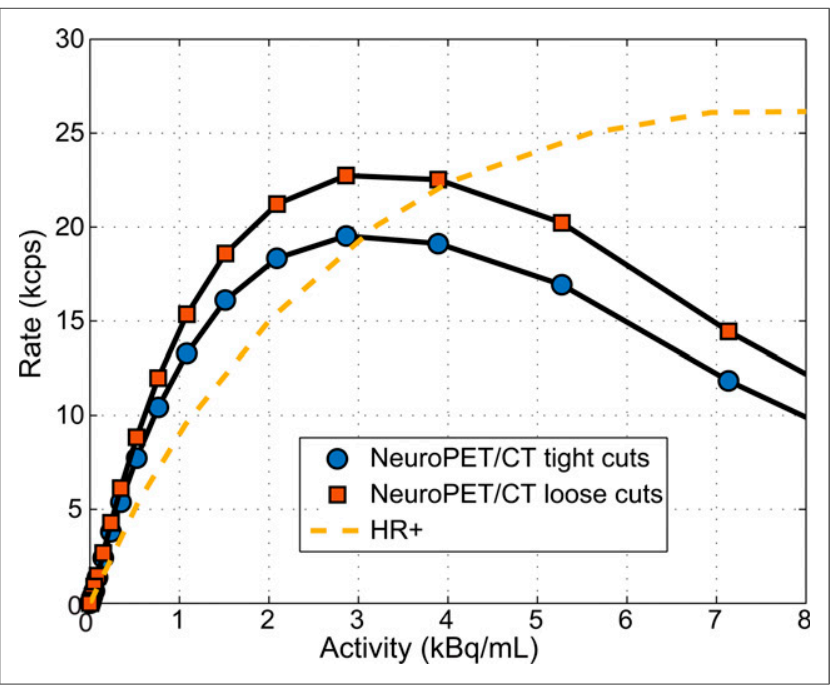

FIGURE 3. NECR versus activity concentration for NeuroPET/CT with tight and loose cuts and for ECAT HR+. 


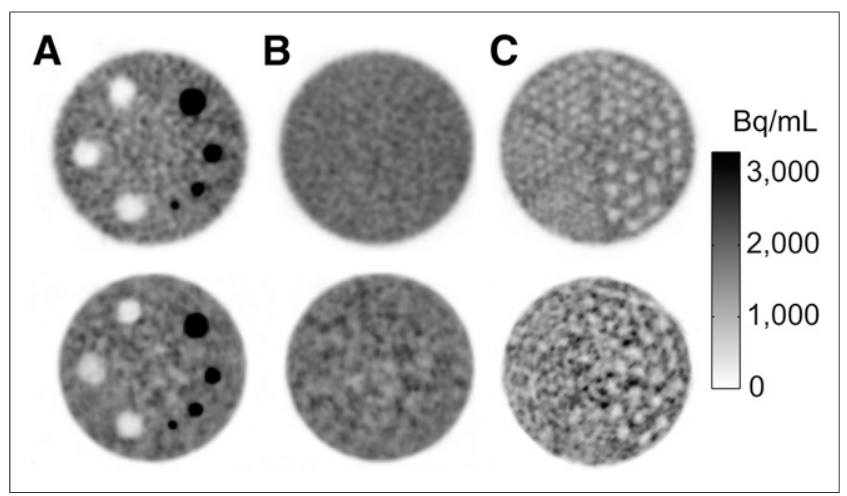

FIGURE 4. NeuroPET/CT (top) and HR+ (bottom) ACR-type phantom showing $10-\mathrm{mm}$ transverse slices of contrast section with cold and hot cylinders (A), uniform section (B), and cold rod section for resolution $(C)$, with second smallest $(6.4 \mathrm{~mm})$ rods resolved for NeuroPET/CT and third largest $(9.5 \mathrm{~mm})$ rods resolved for $\mathrm{HR}+$, although lower resolution in $\mathrm{HR}+$ could be due to proximity to edge of axial FOV. ACR $=$ American College of Radiology.

SF

The mean SF at peak NECR was $42.7 \%$ for the tight cuts and $44.4 \%$ for the loose cuts. This SF is slightly lower than the $46.9 \%$ measured for the HR+ (17).

\section{Dead-Time Correction}

An extrapolation from a linear fit to the system singles at low counting rate was used as an ideal case with no dead time. Relative to the extrapolated fit, the singles rate is undercorrected by $2.4 \%$ at peak NECR and at the typical human ${ }^{18}$ F-FDG singles rates, increasing to $5 \%$ at a rate of 40 Mcps and activity concentration of $7.1 \mathrm{kBq} / \mathrm{mL}$.

\section{Image Quality and Quantitation}

Uniformity Phantoms. The ${ }^{18} \mathrm{~F}$ activity concentration measured in a 4,233-mL ROI in the image was about $2 \%$ higher than the well-counter measurements. There was a slight decrease of the activity toward the edges of the phantom. The coefficient of variation from the grid of the ROI had a mean and SD across the slices of $7.4 \% \pm 1.1 \%$.

American College of Radiology Phantom. Figure 4 shows a transverse view of the hot and cold cylinders, the uniform section, and the resolution using cold rods in a warm background. Rods in 5 of the 6 wedges are resolved. Contrast recovery results are in Table 3 .

Hoffman Brain Phantom. Images from the NeuroPET/CT scan show well-resolved structures and are visually comparable to those of the $\mathrm{HR}+$. Given the differences in reconstruction

\section{TABLE 3}

Contrast Recovery Coefficients for Hot and Cold Vials in American College of Radiology Phantom

\begin{tabular}{ccccccccc}
\hline & \multicolumn{5}{c}{$\begin{array}{c}\text { Cylinder } \\
\text { diameter }(\mathrm{mm})\end{array}$} & & & \\
\cline { 2 - 6 } Cylinder type & 8 & 12 & 16 & 25 & $\mathrm{H}_{2} \mathrm{O}$ & Air & Bone \\
\cline { 2 - 6 } NeuroPET/CT CRC & 0.32 & 0.50 & 0.66 & 0.76 & 0.80 & 0.80 & 0.90 \\
HR+ CRC & 0.44 & 0.61 & 0.61 & 0.73 & 0.67 & 0.63 & 0.72 \\
\hline
\end{tabular}

algorithms, and the need for registration, it is difficult to say that either outperforms the other. Figure 5 shows a slice of the phantom from each scanner.

Human Studies. Human brain images on the NeuroPET/CT were overall of better quality, by visual inspection, than those of the $\mathrm{HR}+$, despite a slightly lower activity concentration. Features of the gray matter were more distinct on the NeuroPET/CT images, although there was slightly more scatter into the white matter regions. An example human brain image is shown in Figure 6 for the HR+ and NeuroPET/CT with $1 \times 1 \times 1.17 \mathrm{~mm}$ voxels. Figure $6 \mathrm{C}$ shows 33 subjects with a global TLS fit and 2 example subjects highlighted. The mutual TLS regression slope of $1.04 \pm 0.02$ is close to 1 . Similarly, the averaged slopes of 33 separate TLS regressions, $1.07 \pm 0.18$, yield a $P$ value of 0.36 , suggesting that data obtained on the NeuroPET/CT are equivalent to those obtained with the HR+.

Animal Scan. PET/CT-fused images of the monkey brain are shown in Figure 7 to demonstrate the capability of the scanner at very low activity levels. Features of the monkey brain are clearly visible in the PET tracer distribution.

\section{DISCUSSION}

The results presented here summarize the imaging capabilities of the NeuroPET/CT, with comparisons to the ECAT HR+. Many of the procedures followed were based on NEMA standards for a fullbody scanner. As such, we did expect some differences in objective results given that the NeuroPET/CT is a head scanner being compared with the full body HR + scanner. Partly for this reason, we also included the results of the human and monkey brain studies.

\section{Spatial Resolution}

Compared with the $\mathrm{HR}+$, the NeuroPET/CT has superior resolution for all point source measurements. The spatial resolution away from the center of the scanner suffers somewhat because of the small ring size. Although the smaller ring provides an advantage in sensitivity over the full-body-sized $\mathrm{HR}+$, it also creates additional uncertainty in the interaction position along the crystal. This disadvantage could be mitigated in the future using depth of interaction information.

\section{Sensitivity}

The NEMA sensitivity is about 1.2 times of that for the HR+ for tight cuts and 1.8 times for loose cuts (more similar to the

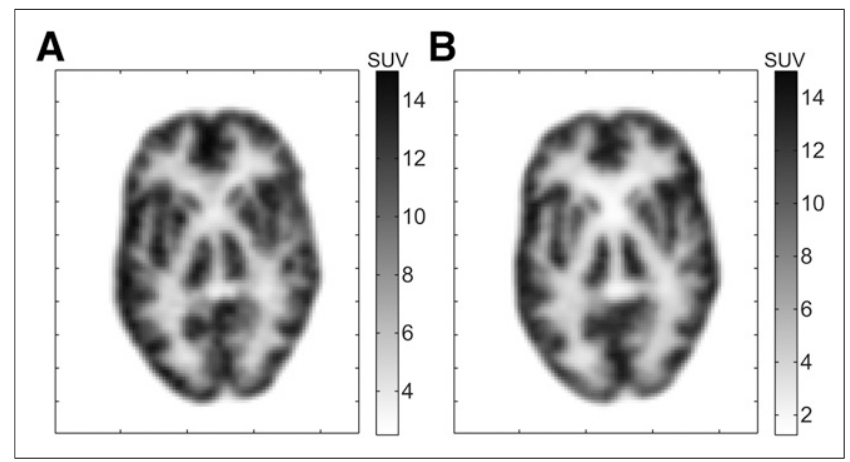

FIGURE 5. Transverse slice from Hoffman phantom for NeuroPET/CT (A) and HR+ (B). SUV is based on 70-kg patient. 


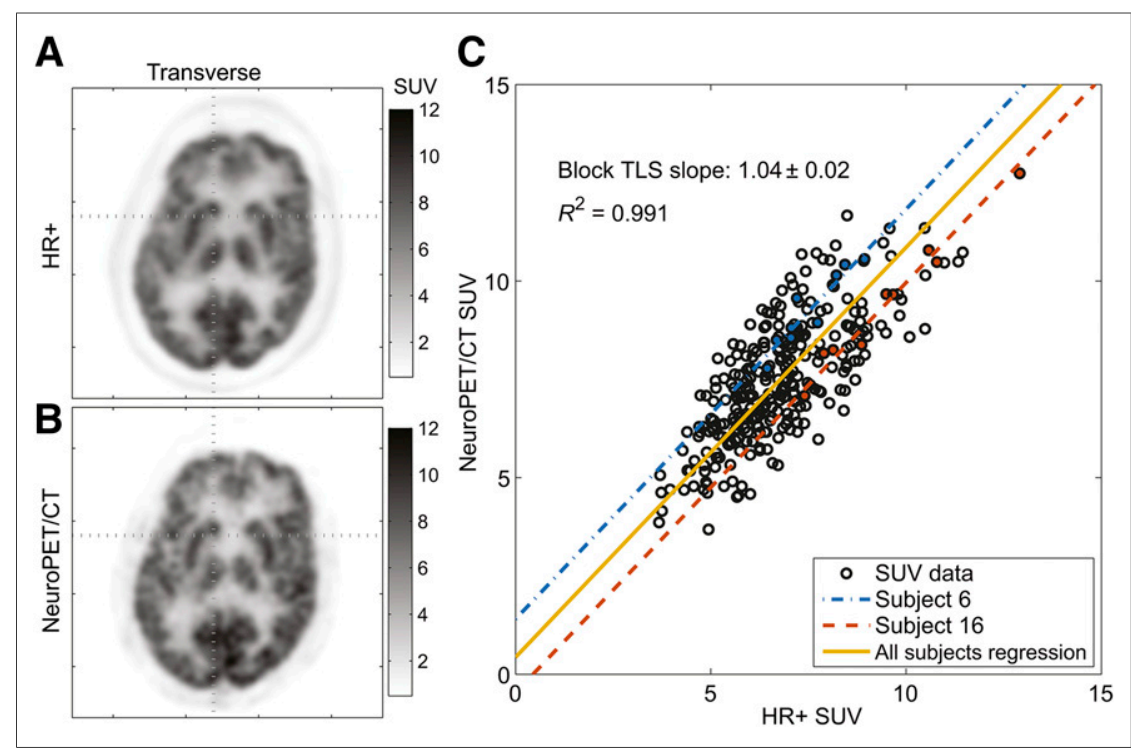

FIGURE 6. ${ }^{18} \mathrm{~F}-\mathrm{FDG}$ images for example subject scanned in $\mathrm{HR}+(\mathrm{A})$ and NeuroPET/CT (B), which show comparable visual quality. (C) Comparison between HR+ and NeuroPET/CT SUV of 9 regions in 33 subjects using blocked total least-squares regression. Two example subjects are shown in blue and red whereas averaged intercept was used for yellow regression line.
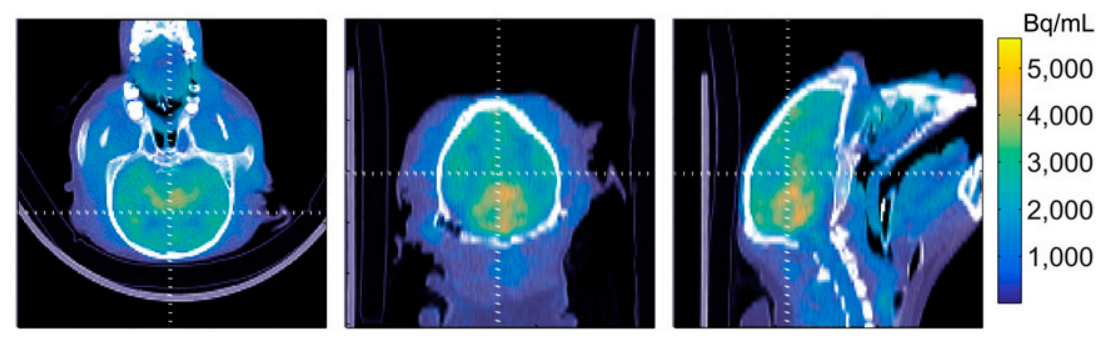

FIGURE 7. Transverse, coronal, and sagittal PET/CT views of monkey brain scanned for $15 \mathrm{~min}$ after injection of $9.25 \mathrm{MBq}(250 \mu \mathrm{Ci})$ of ${ }^{11} \mathrm{C}$ kinase tracer that is under development.

\section{Image Quality and Quantitation}

Image quality and quantitation are suitable for the intended use of the NeuroPET/CT. The contrast recovery is comparable to that of the $\mathrm{HR}+$ - slightly better for the larger cylinders and slightly worse for the smaller cylinders. Currently under investigation is why the NeuroPET/CT, with the better spatial resolution, has worse contrast recovery for the smaller cylinders. There is a high correspondence of SUVs within subjects measured with the $\mathrm{HR}+$ and NeuroPET/CT. Some differences between the human images from the 2 scanners are expected, given the uncertainties from differences in ${ }^{18} \mathrm{~F}-\mathrm{FDG}$ biodistribution, scan time, and registration warping.

\section{CONCLUSION}

The device tested is the first mobile brain PET/CT scanner. In this work, we characterized the performance of the NeuroPET/CT scanner based on NEMA, phantom, and human studies. Our study shows that the scanner has achieved a good combination of performance in terms of spatial resolution, sensitivity, counting rate, and image quality, with the added advantage of mobility and flexibility of use.

\section{DISCLOSURE}

The costs of publication of this article were defrayed in part by the payment of page charges. Therefore, and solely to indicate this fact, this article is hereby marked "advertisement" in accordance with 18 USC section 1734. This work

HR + settings). This higher sensitivity is because NeuroPET/ CT has a large solid angle and smaller gaps between the neighboring modules. The rate was normalized to the full line source of $70 \mathrm{~cm}$. If instead it is normalized to the length of the axial field, $22 \mathrm{~cm}$, then the sensitivity calculation increases by a factor of 3.2, as noted in previous performance studies of head scanners (17).

\section{NECR}

The NECR curve for the NeuroPET/CT peaks at a fairly low activity concentration, but in that region its NECR is 5\%-20\% higher (depending on the cuts used) than the HR+ NECR. The point of peak NECR corresponds to $63 \mathrm{MBq}$ in the line source. That activity corresponds to $19.6 \mathrm{MBq}$, assuming about one third is in the FOV. Although it is somewhat lower than the activity level in a typical ${ }^{18} \mathrm{~F}-\mathrm{FDG}$ scan $(\sim 28 \mathrm{MBq})$, it is not far outside clinical parameters, especially for longer scans. The high NECR at lower activities for the NeuroPET/CT indicates that lower doses can be used without sacrificing imaging quality in terms of signal-to-noise ratio. was supported by NIH grants S10RR028110, T32EB013180, R21EB012823, R01EB019959, and C06CA059267. Terrence Toole is an employee of PDSi. No other potential conflict of interest relevant to this article was reported.

\section{ACKNOWLEDGMENTS}

We thank Zakhar Levin and Steve Weise for their help with phantom and human studies.

\section{REFERENCES}

1. Ishii K. PET approaches for diagnosis of dementia. Am J Neuroradiol. 2014;35:20302038.

2. Basu S, Alavi A. Molecular imaging (PET) of brain tumors. Neuroimaging Clin N Am. 2009;19:625-646.

3. Ouyang J, Keeler M, Bonab A, Zhu X, Brady T, El Fakhri G. Performance measurements of a novel mobile NeuroPET-CT [abstract]. J Nucl Med. 2012;53 (suppl 1):435.

4. Ouyang J, Toole T, Keeler M, et al. Performance comparison between NeuroPET$\mathrm{CT}$ and Siemens ECAT HR+: NEMA and patient studies [abstract]. $\mathrm{J} \mathrm{Nucl}$ Med.2014;55(suppl 1):2162. 
5. National Electrical Manufacturers Association. Performance Measurement of Positron Emission Tomographs. Rosslyn, VA: National Electrical Manufacturers Association; 2012. NEMA standards publication NU 2-2012.

6. Watson CC. New, faster, image-based scatter correction for 3D PET. IEEE Trans Nucl Sci. 2000;47:1587-1594.

7. Bai C, Shao L, Da Silva A, Zhao Z. A generalized model for the conversion from CT numbers to linear attenuation coefficients. IEEE Trans Nucl Sci. 2003;50:1510-1515.

8. Burger C, Goerres G, Schoenes S, Buck A, Lonn AHR, Von Schulthess GK. PET attenuation coefficients from CT images: experimental evaluation of the transformation of CT into PET 511-keV attenuation coefficients. Eur J Nucl Med Mol Imaging. 2002;29:922-927.

9. Defrise M, Kinahan PE, Townsend DW, Michel C, Sibomana M, Newport DF. Exact and approximate rebinning algorithms for 3-D PET data. IEEE Trans Med Imaging. 1997;16:145-158.

10. Daube-Witherspoon ME, Muehllehner G. Treatment of axial data in threedimensional PET. J Nucl Med. 1987;28:1717-1724.

11. Watson CC, Casey ME, Eriksson L, Mulnix T, Adams D, Bendriem B. NEMA NU 2 performance tests for scanners with intrinsic radioactivity. J Nucl Med. 2004;45:822-826.
12. Friston KJ, Holmes AP, Worsley KJ, Poline J-P, Frith CD, Frackowiak RSJ. Statistical parametric maps in functional imaging: a general linear approach. Hum Brain Mapp. 1994;2:189-210.

13. Grabner G, Janke AL, Budge MM, Smith D, Pruessner J, Collins DL. Symmetric atlasing and model based segmentation: an application to the hippocampus in older adults. Med Image Comput Comput Assist Interv. 2006;9:58-66.

14. Deming WE. Statistical Adjustment of Data. New York, NY: Dover Publications; 1964.

15. Adam LE, Zaers J, Ostertag H, Trojan H, Bellemann ME, Brix G. Performance evaluation of the whole-body PET scanner ECAT EXACT HR + following the IEC standard. IEEE Trans Nucl Sci. 1997;44:11721179 .

16. Karakatsanis N, Sakellios N, Tsantilas NX, et al. Comparative evaluation of two commercial PET scanners, ECAT EXACT HR + and Biograph 2, using GATE. Nucl Instrum Methods Phys Res A. 2006;569:368372 .

17. Karp JS, Surti S, Daube-Witherspoon ME, et al. Performance of a brain PET camera based on anger-logic gadolinium oxyorthosilicate detectors. J Nucl Med. 2003;44:1340-1349. 